Check for updates

Cite this: RSC Adv., 2018, 8, 26880

\section{Spherical covalent organic frameworks as advanced adsorbents for preconcentration and separation of phenolic endocrine disruptors, followed by high performance liquid chromatography $\dagger$}

\author{
Jing-Min Liu, (D) a Jia-Li Hao, ${ }^{c}$ Xin-Yue Yuan, ${ }^{b}$ Hui-Lin Liu, ${ }^{b}$ Guo-Zhen Fang (D) ${ }^{c}$ \\ and Shuo Wang*a
}

\begin{abstract}
As a promising generation of porous micro-materials, covalent organic frameworks (COFs) have great potentials for applications in separation and adsorption. In the present study, an advanced food-safety inspection method involving COFs as the adsorbents of solid phase extraction (SPE) is proposed for sensitive and accurate determination of target hazardous substances. Typical spherical TpBD COFs with large surface area and superior chemical stability were utilized as adsorbents for the preconcentration of phenolic endocrine disruptors (PEDs), followed by high performance liquid chromatography (HPLC) analysis. The well-prepared TpBD COFs were encapsulated in SPE cartridges and applied in food research, namely, for the separation and enrichment of four target endocrine disruptors in food samples. The possible factors influencing the SPE performance including the composition of the sample solvent, sample solution $\mathrm{pH}$, sample flow rate, composition of the eluent, and the volume of the eluent were investigated and optimized. Due to the porous architecture and superior surface area of spherical TpBD, the enrichment of analytes via a COF-filled SPE column gave extremely low detection limits of 0.056$0.123 \mu \mathrm{g} \mathrm{L}^{-1}$ along with a wide linear range of $0.5-100 \mu \mathrm{g} \mathrm{L}^{-1}$ for all the analytes. Nine parallel determinations of the mixed standard with a concentration of $10 \mu \mathrm{g} \mathrm{L}^{-1}$ produced the relative standard deviations of $2.23-3.08 \%$, indicating the excellent repeatability of the COF-SPE assay. This study can open up a new route for the employment of COFs as efficient SPE adsorbents for the enrichment and quantification of trace/ultra-trace hazardous materials in complex food samples.
\end{abstract}

Received 21st May 2018 Accepted 9th July 2018

DOI: $10.1039 / \mathrm{c} 8 \mathrm{ra04321c}$

rsc.li/rsc-advances in the industry as important intermediates for the production of polycarbonate plastic and epoxy resins, whereas NP and OP are widely used in the production of plastic packages, elasticizers, abstergents and pesticide emulsifiers. ${ }^{6,7}$ It is worth noting that these phenolic endocrine disruptors widely exist in the environment around us, i.e., in the atmosphere, sediments, soil, environmental water, and food, which can consequently harm human health. Therefore, there is a great demand for rapid, simple, accurate, and sensitive analytical techniques for the monitoring of PEDs in different sample matrices. ${ }^{5,8-12}$ Owing to their trace levels in food and water sample matrices, direct qualification and quantification of PEDs are challenging; hence, an efficient and sensitive sample pre-treatment is urgently required to extract and enrich target PEDs as well as to remove the major interferences prior to instrumental analysis.

As one of the most widely used sample pre-treatment techniques, solid phase extraction (SPE) is favourable for the determination of trace levels of analytes against complex sample matrices due to its rapid phase separation, simplicity, high enrichment factor, low cost, and the variety of efficient 
adsorbents. With the ability of handling large sample volumes, SPE can be easily coupled with diverse analytical instruments via on-line or off-line manner. Generally, the SPE performance largely depends on the employed adsorbent, which is the core and the foundation of preconcentration and enrichment of target analytes. Recently, various SPE adsorbents, usually with large surface areas and porous architectures, such as carbon nanotubes, ${ }^{13,14}$ graphene oxide ${ }^{\mathbf{1 5 , 1 6}}$ metal organic frameworks (MOFs), ${ }^{17}$ mesoporous silica, ${ }^{18}$ and $\mathrm{TiO}_{2}$ nanoparticles ${ }^{19}$ have been developed for specific applications.

As an interesting novel type of porous material, covalent organic frameworks (COFs) possess high-ordered crystalline porous architectures, offering significant potentials for diverse applications in photoelectric, gas storage, separation, and catalysis fields. ${ }^{\mathbf{2 0 - 2 4}}$ Unlike MOFs, which have coordinated structures, COFs are solely fabricated with organic constructing units via strong covalent bonding; therefore, they possess superior chemical stability in acidic media and organic solvents, thus interacting with targets in a wide $\mathrm{pH}$ range. ${ }^{25-27}$ Moreover, due to uniform-sized nanopores and large surface area, COFs exhibit high capacity, fast adsorption, and good regenerability and accessibility. ${ }^{28-31}$ In virtue of the abovementioned observations, we infer that COFs possess great potential as ideal adsorbents for the enrichment of organic compounds via SPE pre-treatment. ${ }^{32-36}$ Very recently, several remarkable studies have well demonstrated COFs as effective adsorbents for polar phenols ${ }^{37-39}$ and other organic pollutants. ${ }^{29,32}$

Herein, typical spherical TpBD COFs, with the advantageous features of large surface area, superior chemical stability, ease of preparation and good dispersity, were utilized as sorbents for the preconcentration of phenolic endocrine disruptors (PEDs), followed by high performance liquid chromatography (HPLC) analysis. The well-prepared TpBD COFs were encapsulated in SPE cartridges and applied in food research, namely, for the separation and enrichment of four target endocrine disruptors in food samples, followed by HPLC measurements. The possible factors influencing the SPE performance including the composition of the sample solvent, sample solution $\mathrm{pH}$, sample flow rate, composition of the eluent, and the volume of the eluent were investigated and optimized. Owing to the porous architecture and superior surface area of spherical TpBD, the enrichment of the analytes via a COF-filled SPE column gave extremely low detection limits of $0.056-0.123 \mu \mathrm{g} \mathrm{L}^{-1}$ along with a wide linear range of $0.5-100 \mu \mathrm{g} \mathrm{L}^{-1}$ for all the analytes. Nine parallel determinations of the mixed standard with a concentration of $10 \mu \mathrm{g} \mathrm{L}^{-1}$ produced the relative standard deviations of $2.23-3.08 \%$, indicating the excellent repeatability of the COFSPE assay. The developed SPE-HPLC method was finally employed for PEDs in food samples.

\section{Experimental section}

\subsection{Chemicals and materials}

All reagents were at least analytical reagent-grade and were used as received. BPA, BPF, OP and NP were purchased from Sigma (Shanghai, China). HPLC-grade acetonitrile and HPLC-grade methanol were obtained from Sinopharm Chemical Reagent Co., Ltd. (Shanghai, China). 1,3,5-Triformylphloroglucinol (Tp) was purchased from Aladdin (Shanghai, China), and benzidine (BD) was purchased from J\&K (Beijing, China). Ethanol and $N, N$ dimethylformamide (DMF) were obtained from Sinopharm Chemical Reagent Co., Ltd. (Shanghai, China). Formic acid and acetic acid were purchased from Aladdin (Shanghai, China). Ultrapure water $\left(18.2 \mathrm{M} \Omega \mathrm{cm}^{-1}\right)$ was generated by the Sartorius Milli-Q system (Aubagne, France) and was used throughout the experiments. All the glassware was cleaned with diluted $\mathrm{HNO}_{3}$ $(10 \%)$ and thoroughly rinsed with ultrapure water prior to use. Mixed standard stock solutions $\left(1000 \mathrm{mg} \mathrm{L}^{-1}\right)$ were prepared by dissolving appropriate amounts of four targets in acetonitrile and storing in dark at $4{ }^{\circ} \mathrm{C}$. Working solutions were obtained daily by diluting the stock solutions with ultrapure water before experiments.

\subsection{Instrumentation}

The microstructure and the morphology of the as-synthesized TpBD were characterized by scanning electron microscopy (SEM) using a LEO 1530VP microscope (LEO, Germany) operated at $10.0 \mathrm{kV}$ and transmission electron microscopy (TEM) using a JEM-2100F field emission transmission electron (JEOL, Japan) microscope at an accelerating voltage of $200 \mathrm{kV}$. The surface area and the porosity of the as-prepared TpBD were measured by nitrogen adsorption-desorption analysis. XRD patterns were acquired on a D/max-2500 diffractometer (Rigaku, Japan) equipped with $\mathrm{Cu} K \alpha$ radiation $(\lambda=1.5418 \AA)$. The contents of the targets were determined with high performance liquid chromatography (HPLC, Shimadzu LC-10, Japan). The standard curve was built according to the target concentrations and the response values.

\subsection{Synthesis of TpBD}

Referring to a previously reported method, ${ }^{32} 15 \mathrm{~mL}$ of ethanol containing $0.30 \mathrm{mmol}$ of $\mathrm{Tp}$ and $5 \mathrm{~mL}$ of ethanol containing $0.45 \mathrm{mmol}$ of BD were mixed in a $100 \mathrm{~mL}$ flask; the solution was continuously stirred for $15 \mathrm{~min}$ at room temperature to obtain a homogenous yellow solution. After evacuation and filling with $\mathrm{N}_{2}$, the reaction system was heated to $80{ }^{\circ} \mathrm{C}$ to reflux under $\mathrm{N}_{2}$ protection, during which the mixed solution demonstrated a clear colour change from yellow to light brown. Then, the reaction mixture was refluxed for another $3 \mathrm{~h}$ under vigorous stirring, and the resultant solution was collected by centrifugation (10 $000 \mathrm{rpm}, 5 \mathrm{~min}$ ). Excess Tp and BD contents were removed by refluxing the obtained solution with DMF for $50 \mathrm{~min}$, followed by refluxing with ethanol for $50 \mathrm{~min}$ to exchange DMF. The resulting products were collected by centrifugation and evacuated under vacuum at $30{ }^{\circ} \mathrm{C}$ overnight to eliminate ethanol. The dried TpBD COFs were finally collected in an agate mortar and ground into a powder to ensure well-dispersion prior to use.

\subsection{SPE procedure}

We used $3 \mathrm{~mL}$ empty SPE cartridges with 2 frits $(8 \mathrm{~mm}$ in diameter, $2 \mu \mathrm{m}$ of pore size) per column as the SPE column; 
$30 \mathrm{mg}$ of the as-synthesized TpBD powder was weighed accurately and added into the SPE column. To avoid collapse, the 2 frits were put on the two sides of the filler.

SPE assay was performed on an SPE vacuum manifold. Three group parallel tests were set for each condition. First, the SPE column was conditioned with $5 \mathrm{~mL}$ of acetonitrile $(10 \%$ acetic acid) and $5 \mathrm{~mL}$ of water. Then, $10 \mathrm{~mL}$ of the working solution was added into the column separately while controlling the flow via a vacuum pump to ensure that the liquid flows slowly through the adsorbent. After loading the sample, the entrapped targets were eluted with $4 \mathrm{~mL}$ of acetonitrile containing $10 \%$ acetic acid. The pooled eluate was evaporated under a gentle stream of nitrogen and reconstituted in $1 \mathrm{~mL}$ of acetonitrile. The solution was filtered through $0.22 \mu \mathrm{m}$ nylon membranes before HPLC analysis.

\subsection{HPLC conditions}

HPLC analysis was performed using Shimadzu LC-10 equipped with a UV-detector. The detection wavelength was $228 \mathrm{~nm}$. Chromatography was performed on an Agilent XDB-C18 $(4.6 \mathrm{~mm} \times 250 \mathrm{~mm}, 5 \mu \mathrm{m})$ column at $35{ }^{\circ} \mathrm{C}$ with an injection volume of $20 \mu \mathrm{L}$. The mobile phase consisting of a solvent A (acetonitrile) and solvent B (water) was delivered at a flow rate of $1 \mathrm{~mL} \mathrm{~min}{ }^{-1}$.

The program was run in a linear gradient:

0-3 min, the proportion of $\mathrm{A}$ in the mobile phase was $60 \%$;

3-15 $\mathrm{min}$, the proportion of $\mathrm{A}$ in the mobile phase increased to $100 \%$, held for $15-17 \mathrm{~min}$;

17-20 min, the proportion of $\mathrm{A}$ in the mobile phase decreased to $60 \%$, held for $20-30 \mathrm{~min}$.

\subsection{Sample pretreatment}

The samples were purchased from a local super-market and stored at $4{ }^{\circ} \mathrm{C}$ before use.

For the beverage samples, certain amounts of standard stock solutions containing the four targets were separately added into the sample solutions to obtain fortified concentrations of the targets as $0,5,10,20 \mu \mathrm{g} \mathrm{L^{-1 }}$. The samples were filtered using $0.22 \mu \mathrm{m}$ pore-size membrane filters before undergoing further analysis. Prior to the TPBD-SPE procedure, the sample solution $\mathrm{pH}$ was adjusted to 4.0 by using $0.1 \mathrm{M}$ formic acid and $0.1 \mathrm{M}$ ammonium hydroxide. The carbonate beverage sample was degassed by ultrasonication for $1 \mathrm{~min}$ in advance.

For the milk sample, the mixtures were spiked using the same procedure as above and were extracted by ultrasonication for $20 \mathrm{~min}$ and centrifugation for $5 \mathrm{~min}$ at $9500 \mathrm{rpm}$. The supernatant was collected and transferred to a flask. The rest was extracted using $20 \mathrm{~mL}$ of acetonitrile again. A rotavapor was used to evaporate the extracted solution to a volume less than 10 $\mathrm{mL}$, which was then evaporated under a gentle stream of nitrogen. Next, it was reconstituted in $1 \mathrm{~mL}$ of acetonitrile and diluted to a fixed volume of $50 \mathrm{~mL}$. The sample solution $\mathrm{pH}$ was also adjusted to 4.0 prior to the SPE-HPLC assay (Fig. 1).

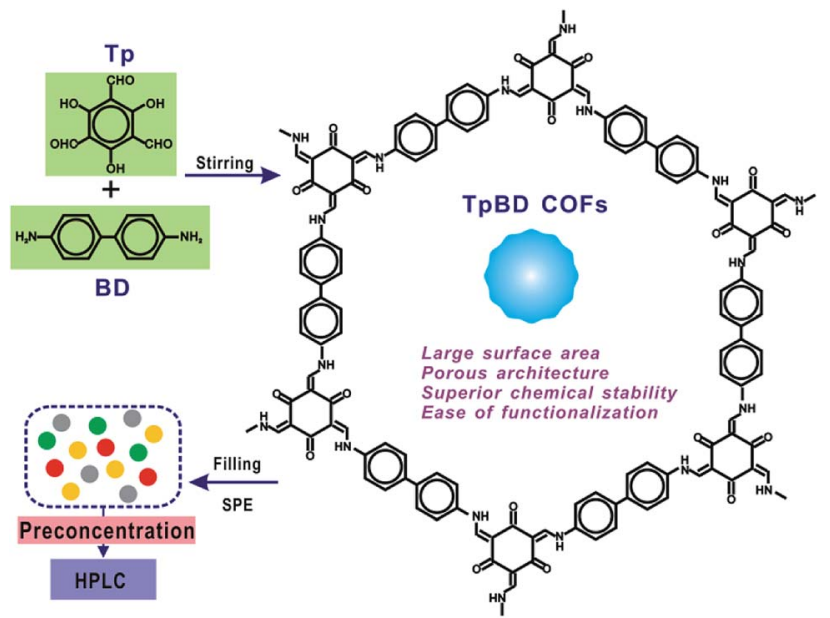

Fig. 1 Schematic illustration of the proposed TpBD-involved SPEHPLC method.

\section{Results and discussion}

\subsection{Synthesis and characterization of TpBD COFs}

As a new generation of porous micro-materials with large surface area and $\pi-\pi$ stacked architecture, TpBD COFs hold great potential for adsorption and separation applications. The key features that qualify TpBD as an outstanding adsorbent are superior chemical stability and unique porous architectures. The XRD pattern (Fig. 2A) revealed the typical crystallinity with a $2 \theta$ peak around the low angle of $3^{\circ}$, which was consistent with the simulated pattern, proving the successful preparation of TpBD. SEM and TEM analyses showed spherical morphology of TpBD with an average particle size of $205 \pm 7 \mathrm{~nm}$. Nitrogen
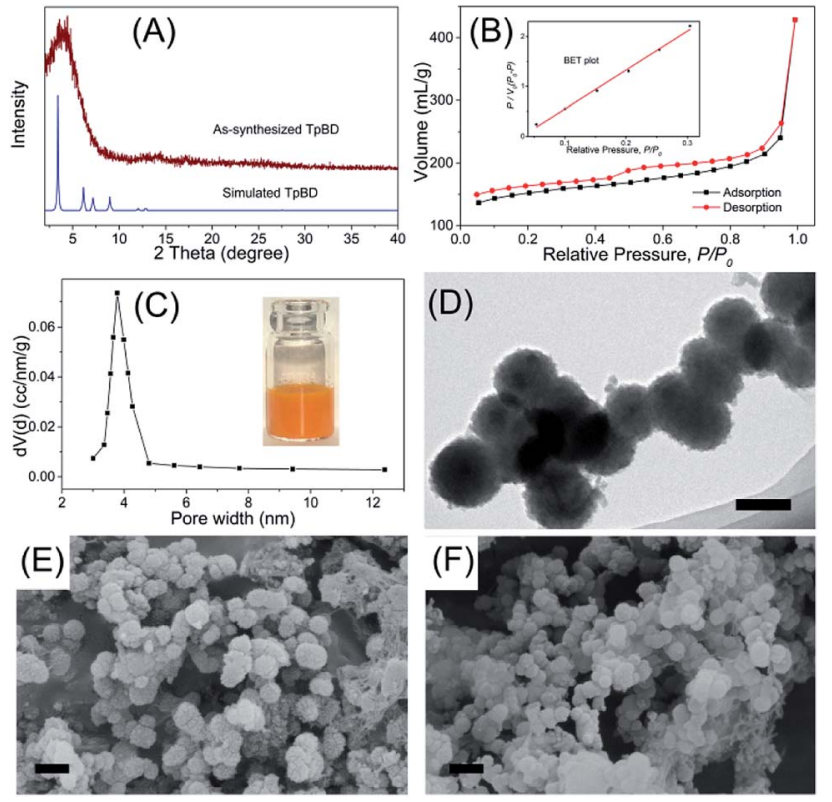

Fig. 2 Characterization of the as-prepared TpBD COFs: (A) XRD analysis; (B) $\mathrm{N}_{2}$ adsorption-desorption isotherm; (C) pore size distribution; (D) a typical TEM image; (E and F) typical SEM images of TpBD before (E) and after (F) adsorption. The scale bars all represent $200 \mathrm{~nm}$. 


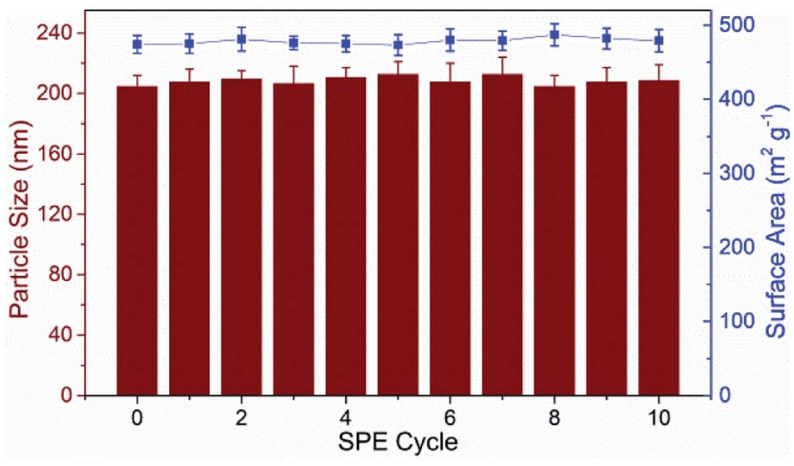

Fig. 3 Continuous monitoring of the surface area and the particle size change of TpBD during the 10 cycles of adsorption/elution.

adsorption-desorption experiments were performed to evaluate the adsorption properties of TpBD. The Brunauer-EmmettTeller (BET) surface area for the as-prepared TpBD was measured to be $474.48 \mathrm{~m}^{2} \mathrm{~g}^{-1}$ with a pore size of $3.8 \mathrm{~nm}$. The large specific surface area demonstrated that the as-prepared TpBD has great potential as an adsorbent for food-borne hazardous substances. SEM results also showed slight changes in the micro-structure of TpBD before and after the SPE-HPLC assay for the preconcentration of target analytes (Fig. 2). Furthermore, slight changes in the surface area and the particle size were observed after 10 cycles of adsorption/elution, indicating good chemical stability of TpBD as an effective adsorbent (Fig. 3).

\subsection{Optimization of TpBD-involved SPE-HPLC assay}

3.2.1 HPLC separation. Different proportions of mobile phases resulted in different separation efficiencies for the four targets, which possessed respective appearance times. In this study, different elution programs with acetonitrile-water as a binary elute were investigated. In program 1 with the mobile phase of $80 \%$ acetonitrile and $20 \%$ water, the appearance times of BPA and BPF were too close to ensure a baseline separation. When the composition of the mobile phase was $60 \%$ acetonitrile $+40 \%$ water, the baseline separation was achieved completely. However, the appearance time of OP was in 2025 min and that of NP was in 65-70 min, which were not suitable for actual operation due to long separation time and poor wide peak shape. Finally, program 3 was conducted in combination with the former two programs as described in the experimental section. Compared with the result for program 2 , the baseline separation was achieved within $20 \mathrm{~min}$ for program 3. Although an upward baseline drift was encountered, possibly due to a change in the proportion of mobile phase during separation, the final separation and quantitative analysis were hardly affected (Fig. 4A). Therefore, the gradient elution of program 3 was applied in chromatographic separation.

3.2.2 Effect of the sample solvent. To examine the effect of the constituents of the sample solvent on the SPE assay, sample solvents with different acetonitrile contents $(0-40 \%)$ were tested upon the preconcentration of the four targets. The results in Fig. 4B show that the SPE recovery decreased significantly when the acetonitrile content was more than $10 \%$, possibly because
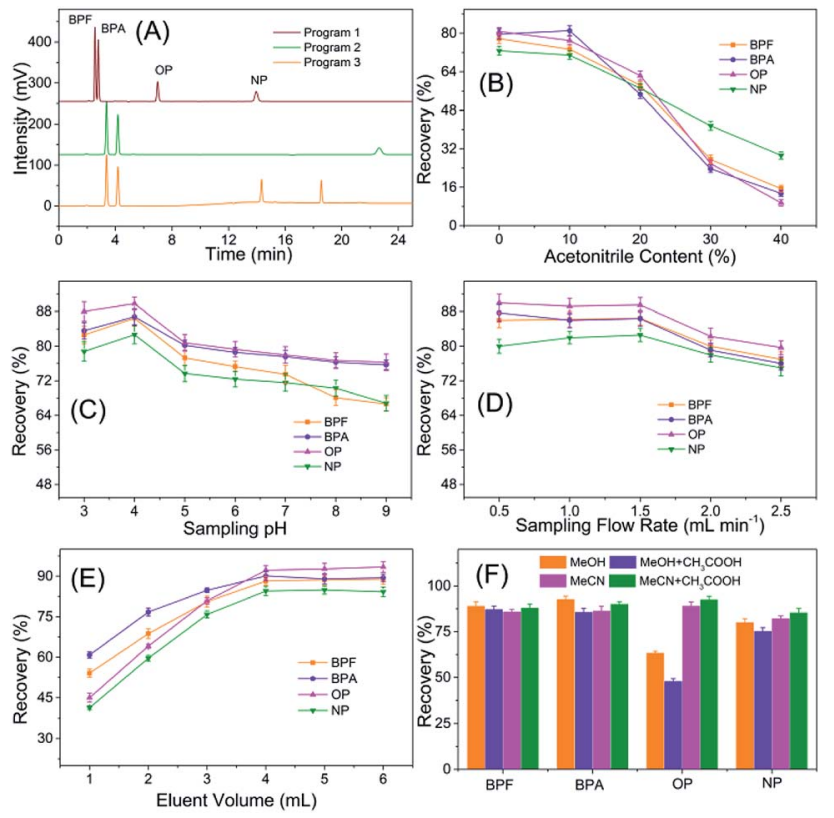

Fig. 4 Optimization of the TpBD-SPE-HPLC assay for analyzing PEDs: (A) HPLC separation conditions; (B) acetonitrile content in the sample solvent; (C) sample solution $\mathrm{pH}$; (D) sample flow rate; (E) eluent volume; (F) elution solvent.

the relatively good solubility of the four targets in acetonitrile affected the interaction of the targets with TpBD. Therefore, the acetonitrile content in the sample solvent was less than $10 \%$ in the following experiments.

3.2.3 Effect of sample solution $\mathrm{pH}$. The $\mathrm{pH}$ of a sample solution always shows great influence on the extraction efficiency, and it directly determines the existing form of chemical species. The effect of $\mathrm{pH}$ on the SPE performance was investigated in the range of 3-9. In low-pH conditions, the recovery increased as the $\mathrm{pH}$ increased, and it reached the maximum at $\mathrm{pH}$ 4. The continual increase of $\mathrm{pH}$ resulted in loss of extraction efficiency (Fig. 4C). Based on the above-mentioned results, $\mathrm{pH}$ 4.0 was chosen as the optimal condition for sample solutions.
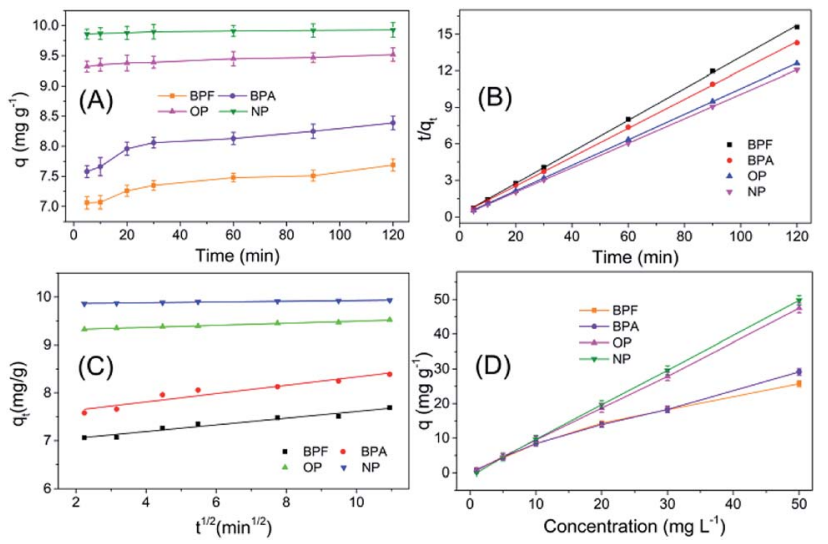

Fig. 5 The adsorption mechanism study of TpBD with the four PBDs: (A) the adsorption kinetics curve of TpBD with the four endocrine disruptors; (B) pseudo-second order adsorption kinetics equation fitting; (C) intra-particle diffusion equation fitting; (D) the adsorption equilibrium experiment of TpBD with the four endocrine disruptors. 
Table 1 Comparison of pseudo-second-order and intra-particle diffusion rate constants for TpBD COF adsorption

\begin{tabular}{lllll}
\hline Target analytes & & BPF & BPA & OP \\
\hline Pseudo-second-order kinetics & $K_{1}\left(\mathrm{~min}^{-1}\right)$ & 0.120 & 0.089 & 0.385 \\
& $q_{\mathrm{e}}\left(\mathrm{mg} \mathrm{g}^{-1}\right)$ & 7.691 & 8.423 & 9.520 \\
& $q_{\mathrm{c}}\left(\mathrm{mg} \mathrm{g}^{-1}\right)$ & 7.584 & 8.346 & 9.433 \\
Intra-particle diffusion & $R^{2}$ & 0.9996 & 0.9996 & 0.9999 \\
& $K_{2}\left(\mathrm{mg} \mathrm{g}^{-1} \mathrm{~min}^{-1 / 2}\right)$ & 0.070 & 0.087 & 0.022 \\
& $C$ & 6.910 & 7.462 & 9.035 \\
& $R^{2}$ & 0.951 & 0.908 & 0.0000 \\
& & & 0.947 \\
& & & 0.959
\end{tabular}

3.2.4 Effect of sample flow rate. Normally, a lower flow rate can increase the interaction time of TpBD with the targets to ensure full adsorption and enrichment but with lower assay efficiency. Herein, the influence of the flow speed on the SPE assay was studied by loading $10 \mathrm{~mL}$ of the working solution of pH 4 through the TpBD-SPE column with a flow rate in the range of $0.5-2.5 \mathrm{~mL} \mathrm{~min}^{-1}$. As shown in Fig. $4 \mathrm{D}$, the SPE recovery showed slight change under low sample flow rates, but it began to decline once the flow rate was increased beyond 1.5 $\mathrm{mL} \min ^{-1}$. Therefore, $1.5 \mathrm{~mL} \min ^{-1}$ was chosen as the optimal loading rate for the subsequent experiments.

3.2.5 Effect of the elution solvent. Elution solvent is critical for the SPE assay, especially to ensure that the analytes are fully eluted from the adsorbing material. In consideration of the solubilities of the analytes, four regular elution media including methanol, methanol containing $10 \%$ acetic acid, acetonitrile and acetonitrile containing $10 \%$ acetic acid were tested on the TpBD-SPE assay. It was found that the four solvents exhibited almost the same extraction efficiencies for BPA and BPF, whereas for OP and NP, acetonitrile containing 10\% acetic acid gave the optimum extraction efficiency, followed by acetonitrile. It should be noted that the recovery of OP was even less than $70 \%$ while using methanol and methanol containing $10 \%$ acetic acid as the elution solvents. In general, the elution abilities of methanol and acetonitrile containing $10 \%$ acetic acid were found to be superior to those of methanol containing $10 \%$ acetic acid and acetonitrile (Fig. 4F). Therefore, acetonitrile containing $10 \%$ acetic acid was chosen for the subsequent experiments.

3.2.6 Effect of the elute volume. In consideration of resources and environmental benefits, the effect of the volume of the elution solvent used was carefully assessed to reduce the amount as much as possible on the premise that the analyte can be fully eluted from the adsorbing material. It can be observed

Table 2 Analytical performance of the TpBD-SPE-HPLC method

\begin{tabular}{|c|c|c|c|c|c|c|}
\hline \multirow[b]{2}{*}{ PEDs } & \multirow[b]{2}{*}{$\begin{array}{l}\text { Linear range } \\
\left(\mu g \mathrm{~L}^{-1}\right)\end{array}$} & \multirow[b]{2}{*}{$R^{2}$} & \multirow[b]{2}{*}{$\begin{array}{l}\text { LOD } \\
\left(\mu g \mathrm{~L}^{-1}\right)\end{array}$} & \multirow[b]{2}{*}{$\begin{array}{l}\text { LOQ } \\
\left(\mu g \mathrm{~L}^{-1}\right)\end{array}$} & \multicolumn{2}{|c|}{ RSD (\%) } \\
\hline & & & & & $\begin{array}{l}\text { intra } \\
n=9\end{array}$ & inter $n=7$ \\
\hline BPF & $0.25-100$ & 0.9993 & 0.056 & 0.185 & 2.33 & 4.35 \\
\hline BPA & $0.25-100$ & 0.9993 & 0.063 & 0.208 & 2.82 & 4.86 \\
\hline $\mathrm{OP}$ & $0.5-100$ & 0.9909 & 0.103 & 0.340 & 3.08 & 3.97 \\
\hline NP & $0.5-100$ & 0.9954 & 0.122 & 0.403 & 2.23 & 4.62 \\
\hline
\end{tabular}

from Fig. 4E that the recovery increased as the volume increased in the range of 1-4 $\mathrm{mL}$ and remained almost unchanged with the further increase in the volume. Four $\mathrm{mL}$ was the minimum volume of the elution solvent that could effectively elute the analyte in TpBD-SPE; thus, it was chosen to be used throughout the experiments.

\subsection{Adsorption mechanism study}

The adsorption behaviour of TpBD with the four target analytes was systemically studied to probe the possible mechanism of adsorption. The adsorption kinetic curves exhibited a relatively rapid adsorption process, and all the adsorptions reached equilibrium within $30 \mathrm{~min}$, along with a satisfactory adsorption capacity, which was suitable for SPE application (Fig. 5A).

For the purpose of probing the possible mechanism of adsorption behaviours in this assay, pseudo-second-order and intra-particle diffusion models have been introduced to interpret the experimental data. The rate constants for the two models have been determined, and the correlation coefficients have been calculated to assess the model providing the best fit of the predicted data with the experimental results. It is found that the pseudo-second-order equation provides a better $R^{2}$ value for the linear fitting and a good agreement between the calculated $q_{\mathrm{c}}$ values and the experimental $q_{\mathrm{e}}$ values, whereas the intra-particle diffusion equations do not give a good fit to the experimental data for the adsorption (Fig. 5B and C, Table 1).

In the following adsorption equilibrium experiment, the adsorption capacities of the four targets continually increased as the concentration increased in the range of $1-50 \mathrm{mg} \mathrm{L}^{-1}$,

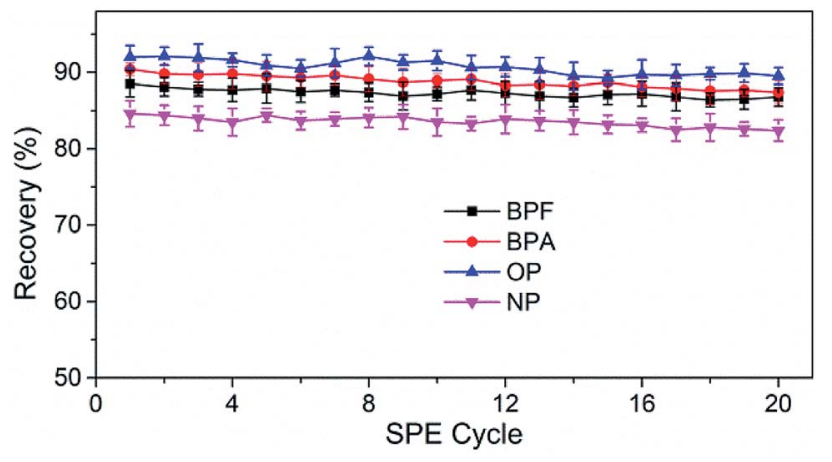

Fig. 6 Evaluation of the reusability of TpBD-packed SPE columns for PED analysis. 
Table 3 Analytical results of the food sample analysis using the presented TpBD-involved SPE-HPLC method (mean \pm SD, $n=3$ )

\begin{tabular}{|c|c|c|c|c|}
\hline Targets & & Spiked $\left(\mu \mathrm{g} \mathrm{L}{ }^{-1}\right)$ & $\begin{array}{l}\text { Detection value } \\
(\text { mean } \pm \mathrm{SD})\left(\mu \mathrm{g} \mathrm{L}^{-1}\right)\end{array}$ & Recovery (\%) \\
\hline \multirow[t]{16}{*}{ Carbonate beverage } & \multirow[t]{4}{*}{$\mathrm{BPF}$} & 0 & $1.35 \pm 0.07$ & - \\
\hline & & 5 & $5.86 \pm 0.24$ & 90.2 \\
\hline & & 10 & $10.17 \pm 0.11$ & 88.2 \\
\hline & & 20 & $19.62 \pm 0.10$ & 91.4 \\
\hline & \multirow[t]{4}{*}{ BPA } & 0 & $1.89 \pm 0.06$ & - \\
\hline & & 5 & $6.33 \pm 0.07$ & 88.8 \\
\hline & & 10 & $11.17 \pm 0.14$ & 92.8 \\
\hline & & 20 & $19.91 \pm 0.11$ & 90.1 \\
\hline & \multirow[t]{4}{*}{$\mathrm{OP}$} & 0 & - & - \\
\hline & & 5 & $4.63 \pm 0.13$ & 92.6 \\
\hline & & 10 & $9.41 \pm 0.16$ & 94.1 \\
\hline & & 20 & $18.08 \pm 0.12$ & 90.4 \\
\hline & \multirow[t]{4}{*}{$\mathrm{NP}$} & 0 & - & - \\
\hline & & 5 & $4.25 \pm 0.08$ & 85.0 \\
\hline & & 10 & $8.74 \pm 0.24$ & 87.4 \\
\hline & & 20 & $17.98 \pm 0.15$ & 89.9 \\
\hline \multirow[t]{16}{*}{ Beverage } & \multirow[t]{4}{*}{ BPF } & 0 & $1.21 \pm 0.08$ & - \\
\hline & & 5 & $5.46 \pm 0.10$ & 85.0 \\
\hline & & 10 & $9.92 \pm 0.07$ & 87.1 \\
\hline & & 20 & $19.19 \pm 0.19$ & 89.9 \\
\hline & \multirow[t]{4}{*}{$\mathrm{BPA}$} & 0 & $2.15 \pm 0.07$ & - \\
\hline & & 5 & $6.61 \pm 0.04$ & 89.2 \\
\hline & & 10 & $11.27 \pm 0.18$ & 91.2 \\
\hline & & 20 & $20.83 \pm 0.20$ & 93.4 \\
\hline & \multirow[t]{4}{*}{$\mathrm{OP}$} & 0 & - & - \\
\hline & & 5 & $4.62 \pm 0.09$ & 92.4 \\
\hline & & 10 & $9.04 \pm 0.13$ & 90.4 \\
\hline & & 20 & $17.98 \pm 0.18$ & 89.9 \\
\hline & \multirow[t]{4}{*}{ NP } & 0 & - & - \\
\hline & & 5 & $4.17 \pm 0.12$ & 83.4 \\
\hline & & 10 & $8.61 \pm 0.10$ & 86.1 \\
\hline & & 20 & $17.44 \pm 0.05$ & 87.2 \\
\hline \multirow[t]{16}{*}{ Milk } & \multirow[t]{4}{*}{$\mathrm{BPF}$} & 0 & $2.09 \pm 0.04$ & - \\
\hline & & 5 & $6.60 \pm 0.13$ & 90.2 \\
\hline & & 10 & $11.45 \pm 0.09$ & 93.6 \\
\hline & & 20 & $20.45 \pm 0.11$ & 91.8 \\
\hline & \multirow[t]{4}{*}{ BPA } & 0 & $2.36 \pm 0.07$ & - \\
\hline & & 5 & $6.94 \pm 0.05$ & 91.6 \\
\hline & & 10 & $11.59 \pm 0.12$ & 92.3 \\
\hline & & 20 & $20.04 \pm 0.16$ & 88.4 \\
\hline & \multirow[t]{4}{*}{$\mathrm{OP}$} & 0 & - & - \\
\hline & & 5 & $4.58 \pm 0.10$ & 91.6 \\
\hline & & 10 & $9.63 \pm 0.13$ & 96.3 \\
\hline & & 20 & $18.96 \pm 0.09$ & 94.8 \\
\hline & \multirow[t]{4}{*}{$\mathrm{NP}$} & 0 & $1.45 \pm 0.02$ & - \\
\hline & & 5 & $5.55 \pm 0.06$ & 82.0 \\
\hline & & 10 & $9.81 \pm 0.10$ & 83.6 \\
\hline & & 20 & $18.73 \pm 0.09$ & 86.4 \\
\hline
\end{tabular}

demonstrating that TpBD COFs possessed robust adsorption capability for the targets. The adsorption process was interpreted with both Freundlich and Langmuir isotherm models, and the comparison results showed that the calculated data were better fitted with the Freundlich model than with the Langmuir model, revealing that the adsorption was a multiple process rather than a monolayer adsorption process. The Freundlich constant $(n)$ values of the four target PBDs were all $>1$, which indicated the favorable adsorption of the analytes by TpBD.
The adsorption capability has been compared with that of a commercial SPE column (Cleanert PEP, Bonna-Agela Technologies, Tianjin, China) filled with polyethylene vinyl benzene microspheres $(40-60 \mu \mathrm{m})$ and the other two types of COFs TpPa-1 and TpPa-2. The results in Fig. S1 $\dagger$ indicate that the SPE performance of TpBD to the four targets was better than that of the commercial PEP column and comparable with those of TpPa-1 and TpPa-2 in terms of the adsorption capacity. 


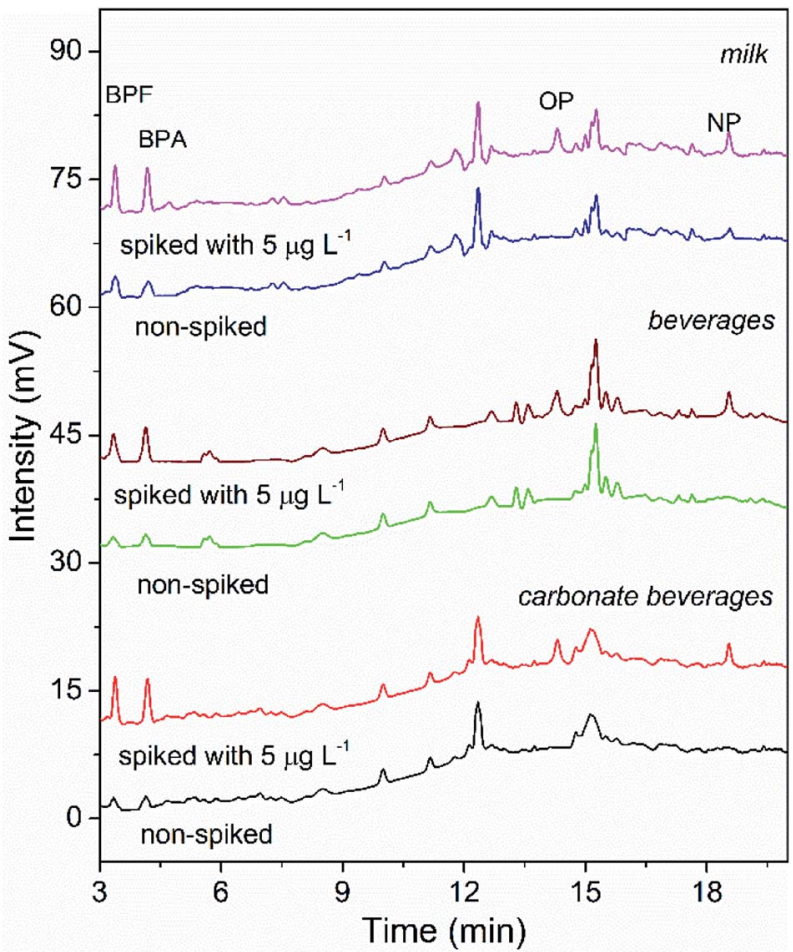

Fig. 7 HPLC chromatograms of the real sample analysis using the developed COF-SPE method (non-spiked and spiked with $5 \mu \mathrm{g} \mathrm{L}^{-1}$ target analytes).

\subsection{Analytical performance of the COF-SPE-HPLC assay}

Using the optimized experimental conditions, calibration curves of the analytes were obtained at concentrations between 0.5 and $100 \mu \mathrm{g} \mathrm{L}^{-1}$. The equations of linear regression, correlation coefficients $\left(R^{2}\right)$ and detection limits were obtained from the calibration curves. The relative standard deviation (RSD) values were obtained using nine determinations and seven determinations of $10 \mu \mathrm{g} \mathrm{L}^{-1}$ target solutions for intra-day and inter-day precision, respectively. As can be seen from Table 2, the $R^{2}$ values of all the analytes were greater than 0.99, and those of BPA and BPF were even higher than 0.999. RSDs of the analytes ranged from 2.23 to $3.08 \%$. The results showed that the method has good stability and precision, which indicated that the enrichment column with TpBD-SPE was suitable for the determination of BPA, BPF, OP, and NP. The detection limits of BPA, BPF, OP, and NP were 0.056, $0.063,0.103$, and $0.123 \mu \mathrm{g} \mathrm{L}^{-1}$, respectively, which were far less than the prescriptive quantity or the transfer quantity values, demonstrating that the proposed method can satisfy the examination requirement of trace PEDs in food.

The reusability and stability of TpBD were tested by spacing and non-spacing adsorption and elution cycles, and the column efficiency was evaluated by observing the changes in the capacities of the analytes. Although there was a declining trend for the recovery of analytes with the increase in extraction times, the change was slight, and the recoveries of the analytes were all above $80 \%$ after 20 adsorption-elution cycles, proving the stability of TpBD COFs in the SPE cycle tests and good reusability of the developed TpBD-packed SPE columns for HPLC methods (Fig. 6).

\subsection{Real sample analysis}

To validate the suitability of the presented COF-SPE-HPLC assay, it was applied to analyze the targets in beverage and milk samples. Low concentrations of BPA and BPF were found in all samples, and trace levels of NP were detected in the milk sample. Fifty $\mathrm{mL}$ of three samples spiked with 5,10 and $20 \mu \mathrm{g}$ $\mathrm{L}^{-1}$ of each analyte was extracted by the TpBD-SPE assay. The recoveries of the spiked samples (Table 3 ) were obtained in the range of $85.0-94.1 \%$ for carbonate beverage samples, 83.493.4\% for regular beverage samples and $82.0-96.3 \%$ for milk samples. The precision (RSD) was in the range of $0.5-6.6 \%$. Fig. 7 shows the chromatograms of the three samples spiked with $5 \mu \mathrm{g} \mathrm{\textrm {L } ^ { - 1 }}$ mixed standards. All of these results confirmed the applicability of the proposed TpBD-SPE-HPLC method for food sample analysis.

\section{Conclusions}

In the present study, a novel spherical TpBD COF was successfully prepared and employed as the adsorbent and preconcentration material for SPE, followed by HPLC for the separation and quantification of PEDs. The superior chemical stability and excellent dispersity of TpBD guaranteed more than 20 adsorption-elution cycles with acceptable recoveries. The proposed method was proven to be rapid, reliable and highly sensitive. This study can open up a new route for the introduction of COFs as efficient SPE adsorbents for the preconcentration and determination of trace/ultra-trace hazardous substances in complex food samples.

\section{Conflicts of interest}

There are no conflicts to declare.

\section{Acknowledgements}

This work was supported by National Key R\&D Program of China (No. 2018YFD0400703), the Fundamental Research Funds for the Central Universities, the Science and Technology Program of Tianjin, China (project No. 17ZYPTJC00050), and China Postdoctoral Science Foundation (No. 2017M621059).

\section{Notes and references}

1 M. Wu, X. Guo, F. Zhao and B. Zeng, Sci. Rep., 2017, 7, 8912. 2 S. Ehrlich, P. L. Williams, S. A. Missmer, J. A. Flaws, K. F. Berry, A. M. Calafat, X. Ye, J. C. Petrozza, D. Wright and R. Hauser, Environ. Health Perspect., 2012, 120, 978-983.

3 L. Yang, Z. Li, L. Zou and H. Gao, Chemosphere, 2011, 83, 233-239.

4 M. K. R. Mudiam, R. Jain and R. Singh, Anal. Methods, 2014, 6, 1802-1810.

5 L. Liu, T. Xia, X. Zhang, D. B. Barr, A. Alamdar, J. Zhang, M. Tian, Q. Huang and H. Shen, Anal. Bioanal. Chem., 2014, 406, 5049-5054. 
6 Y.-n. Dai, A. Dan, Y. Yang, N. F.-y. Tam, Y.-P. Tai and X.-Y. Tang, Environ. Sci. Technol., 2016, 50, 11844-11852.

7 Z. Li, H. Zhang, M. Gibson and J. Li, Toxicol. In Vitro, 2012, 26, 769-774.

8 R. Penu, A. C. Obreja, D. Patroi, M. Diaconu and G. L. Radu, Microchem. J., 2015, 121, 130-135.

9 H. Gallart-Ayala, E. Moyano and M. T. Galceran, Mass Spectrom. Rev., 2010, 29, 776-805.

10 L. Aguei, V. Serafin, P. Yanez-Sedeno and J. M. Pingarron, Anal. Methods, 2010, 2, 499-506.

11 Y. C. Fiamegos and C. D. Stalikas, Anal. Chim. Acta, 2007, 597, 32-40.

12 R. B. Pellegrino Vidal, G. A. Ibanez and G. M. Escandar, $R S C$ Adv., 2015, 5, 20914-20923.

13 H. Peng, N. Zhang, M. He, B. Chen and B. Hu, Talanta, 2015, 131, 266-272.

14 P. Liang, Y. Liu, L. Guo, J. Zeng and H. Lu, J. Anal. At. Spectrom., 2004, 19, 1489-1492.

15 K. Pytlakowska, V. Kozik, M. Matussek, M. Pilch, B. Hachula and K. Kocot, RSC Adv., 2016, 6, 42836-42844.

16 A. Islam and S. Kumar, RSC Adv., 2016, 6, 77629-77635.

17 Z.-Y. Gu, C.-X. Yang, N. Chang and X.-P. Yan, Acc. Chem. Res., 2012, 45, 734-745.

18 P. Li, X.-q. Zhang, Y.-j. Chen, T.-y. Bai, H.-z. Lian and X. Hu, RSC Adv., 2014, 4, 49421-49428.

19 Y. Zhang, C. Zhong, Q. Zhang, B. Chen, M. He and B. Hu, RSC Adv., 2015, 5, 5996-6005.

20 N. Huang, X. Chen, R. Krishna and D. Jiang, Angew. Chem., Int. Ed., 2015, 54, 2986-2990.

21 W. Li, C.-X. Yang and X.-P. Yan, Chem. Commun., 2017, 53, 11469-11471.

22 H.-L. Qian, C.-X. Yang and X.-P. Yan, Nat. Commun., 2016, 7, 12104.
23 C. Mongin, S. Garakyaraghi, N. Razgoniaeva, M. Zamkov and F. N. Castellano, Science, 2016, 351, 369-372.

24 Y. Zeng, R. Zou, Z. Luo, H. Zhang, X. Yao, X. Ma, R. Zou and Y. Zhao, J. Am. Chem. Soc., 2015, 137, 1020-1023.

25 S. He, T. Zeng, S. Wang, H. Niu and Y. Cai, ACS Appl. Mater. Interfaces, 2017, 9, 2959-2965.

26 W. Xuan and Y. Nengsheng, Electrophoresis, 2017, 38, 30593078.

27 Y. Wen, L. Chen, J. Li, D. Liu and L. Chen, TrAC, Trends Anal. Chem., 2014, 59, 26-41.

28 F. L. Zhao, H. M. Liu, S. D. R. Mathe, A. J. Dong and J. H. Zhang, Nanomaterials, 2018, 8, 15.

29 H. L. Qian, C. X. Yang, W. L. Wang, C. Yang and X. P. Yan, J. Chromatogr. A, 2018, 1542, 1-18.

30 J. L. Segura, M. J. Mancheno and F. Zamora, Chem. Soc. Rev., 2016, 45, 5635-5671.

31 N. Huang, P. Wang and D. L. Jiang, Nat. Rev. Mater., 2016, 1, 16068.

32 C.-X. Yang, C. Liu, Y.-M. Cao and X.-P. Yan, Chem. Commun., 2015, 51, 12254-12257.

33 B. Yang, J. Bjork, H. Lin, X. Zhang, H. Zhang, Y. Li, J. Fan, Q. Li and L. Chi, J. Am. Chem. Soc., 2015, 137, 4904-4907.

34 S.-Y. Ding and W. Wang, Chem. Soc. Rev., 2013, 42, 548-568.

35 X. Feng, X. Ding and D. Jiang, Chem. Soc. Rev., 2012, 41, 6010-6022.

36 A. P. Cote, A. I. Benin, N. W. Ockwig, M. O'Keeffe, A. J. Matzger and O. M. Yaghi, Science, 2005, 310, 1166-1170.

37 J.-Y. Ren, X.-L. Wang, X.-L. Li, M.-L. Wang, R.-S. Zhao and J.-M. Lin, Anal. Bioanal. Chem., 2018, 410, 1657-1665.

38 Q.-L. Li, F. Huang, X.-L. Wang, X. Wang and R.-S. Zhao, Microchim. Acta, 2017, 184, 1817-1825.

39 W.-K. Meng, L. Liu, X. Wang, R.-S. Zhao, M.-L. Wang and J.-M. Lin, Anal. Chim. Acta, 2018, 1015, 27-34. 